\title{
Clue Tables: A Naming Mechanism for Building Highly Available and Scalable Distributed File Systems
}

\author{
Cheng-Zen Yang and Yen-Jen Oyang \\ Department of Computer Science and Information Engineering \\ National Taiwan University \\ Taipei, Taiwan, China
}

\begin{abstract}
The naming mechanism plays an important role in a distributed file system. It is one of the determinant factors that affect the availability, scalability, and management work of the distributed system. This paper proposes a new naming mechanism called clue tables. The clue table mechanism offers all the desirable features of high availability, high scalability, and simple procedure for expanding file server capacity.
\end{abstract}

\section{Introduction}

The naming mechanism in a distributed file system maps the logical name of a file into its physical location. The main issue in the design of a naming mechanism is to provide a unique, distribution-transparent mapping for each file. The existing naming mechanismis can be classified into two categories: centralized and distributed. In a centralized naming mechanism such as the mechanism in Locus $[1,2]$, a centralized name server is designated to take the responsibility of mapping the logical file name into physical file location. Apparently, the centralized approach suffers the drawback of low scalability and availability. The centralized name server has the potential to become the system bottleneck and thus limit the scalability of the system because it is a unique resource shared by all the clients. The availability of a system with a centralized naming server is low too because in event of failure of the naming server the entire system is out of service.

Another category of naming mechanisms involves distribution of the naming operations to multi- ple agents in the systen. Due to the distributed nature, the system no longer suffers low availability and scalability as with a centralized mechanism. Some systems with distributed naming employ static binding while some others employ dynamic binding. NFS [3] and Ficus $[4,5]$ are two examples of distributed naming with static binding. In such a system, a remote file system nust be determinately mounted at the system startup time. The problem with this approach is that it makes the expansion and management of a large system cumbersome and tedious. Sprite [6], AFS [7], and Deceit [8] are some examples of distributed naming with dynamic binding. In such a system, the naming operation is conducted either through lookup in a distributed name table or by the decentralized servers. Apparently, distributed naming with dynamic binding is advantageous over previous two approaches.

In this paper, we propose a new naming mechanisiu called clue tables. The clue table mechanism is basically a distributed naning with dynamic binding mechanism. If compared with other distributed naming with dynamic binding mechanisms, clue tables offer all the desired features of high availability, high scalability, and simple procedure for expanding file server capacity. A distributed system with clue tables enjoys high availability because replicated copies of a file co-exist in a number of file servers and each copy is essentially accessible to all the clients in the system. As a result, the operation of the system will not discontinue due to the failure of a single file server. The scalability of a distributed system with clue tables is high because concurrent accesses to a file can be served by multiple servers that have a replicated copy of the file. The only concern with concurrent 
file accesses is that data consistence must be maintained throughout the system. In event one of the concurrent accesses involve writing, some overhead is inevitable for maintaining data consistence. Fortunately, according to a previous study [9], this situation seldomly occurs and therefore will not cause significant performance degradation. In a distributed system with clue tables, the expansion of a file server capacity involves just adding new disks to the server. No update to the contents of the clue tables is needed.

In the following part of the paper, we will present the scenario of clue tables in section 2 , and summerize our discussion in Section3.

\section{Clue Tables}

Before moving to the discussion of clue tables, let us first define the term domain. A domain is the integrated file system in a distributed system. Each file in a domain is an object of two attributes: logical file name and physical locations.

\subsection{Basic Structure}

Fig. 1 shows an example of clue tables. A clue table contains a number of entries, each of which corresponds to a directory in the domain. For example, in Fig. 1, there are two entries. The first entry is corresponding to root directory " $P$ " and the second entry is corresponding to directory "/usr". A clue table entry specifies the file servers that have a replicated copy of the directory. For example, the root directory has two copies stored in file servers solar and earth, respectively. By having multiple copies of a file, the availability of the system is significantly enhanced.

A clue table entry is overridden by another entry when the second directory is a specifier of one of its subdirectories. For example, in Fig. I, the second entry, corresponding to direcotry "/usr", overrides the first entry. When a client tries to locate a file, it first searches the clue tables for the longest prefix of the directories that match the filename. The client then sends a request to the first server in the name list. The server responds to the request by locating the file in its own storage and retuming a succeeded or failed message. If the first server in the name list does not respond within a predetermined period, then the client goes after the second server in the name list and so forth

\subsection{Global and Local Clue Tables}

Initially, a distributed system has only one global clue table which is created when the system is set up. Once the system is up and running, each client can creat its own local clue table by making a copy of the global chue table and making some changes to the local copy. The changes that a client most likely makes are reordering the servers in the name list of a clue table entry. With different orders in the name lists, two clients then will normally approach different servers while accessing the same file. This, in turn, will avoid forming bottlenecks in the system and thus increase system scalability.

The local clue table can be stored in a client's local disk if it has one. If the client is diskless, then the local clue table will be a regular file that is cached in the client's main menory while it is operating.

\subsection{Clue Aliasing}

A clue aliasing mechanismu is incorporated to provide more flexibility in system integration. The second entry in Fig. 1 shows an example of clue aliasing. When the client accesses the directory /usr and sends a request to server csm, it is actually accessing the directory /usr/sparc on csm. Through clue aliasing, a directory on a server can appear in different names on different clients. This adds desirable flexibility to system integration.

\subsection{Private Naming Space}

In a distributed system, some clients may have their own local disks. In such case, the clients can always put the local file system at the first place in the name lists of the local clue table entries. By doing this, the client will always search its local file system when locating a file. For security reason, the client may limit the access previlege of the local file system to remote requestors 


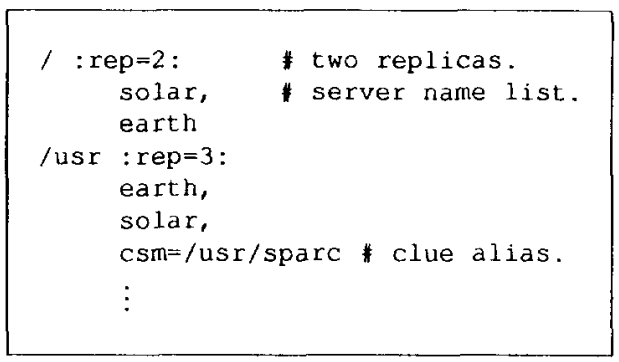

Figure 1: An example of global clue table and clue aliases

\subsection{Expansion of File Server Capacity}

From time to time, new disks will be added to the file servers of a distributed system. With clue tables, the only procedure that the system manager needs to take to expand file server capacity is just to complete hardware installation of the new disks on file servers. There is no need to update the contents of the clue tables due to the dynanic binding nature of clue tables. This makes the procedure extremely simple and the system easy to maintain.

\section{Conclution}

In this paper, we proposed a new naming mechanism called clue tables for distributed systems. The clue table mechanism is distinctive from existing naming mechanisns by offering all the desirable features of high availability, high scalability, and simple procedure for expanding file server capacity. Distributed systems with clue tables are highly available because replicated copies of a file co-exist on multiple servers and thus the operation of the system will not discontinue due to the failure of a single file server. Such systems are highly scalable because concurrent accesses to a file can be serviced by multiple servers that have a replicated copy of the file. Finally, it is extremely easy to expand the file server capacity in a distributed system with clue tables. What the system manager really needs to do is just to complete the hardware installation of the new disks.

\section{References}

[1] G. Popek, B. Walker, J. Chow, D. Edwards, C. Kline, G. Rudisin, and G. Thiel. "LOCUS: A Network Transparent, High Reliability Distributed System". In Proc. of 8th Symposium Operating Systems Principles, pages 169-177. ACM, Dec. 1981.

[2] B. Walker, G. Popek, R. English, C. Kline, and G. Thiel. "The LOCUS Distributed Operating System". In Proc. of 9th Symposium Operating Systems Principles, pages 49-70. ACM, Oct. 1983.

[3] R. Sandberg, D. Goldberg, S. Kleiman, D. Walsh, and B. Lyon. "Design and Inplementation of the Sun Network File System". In USENIX Conference proceedings, pages 119-130, June 1985.

[4] R. G.Guy, J. S. Heidentann, W. Mak, T. W. Page, G. J. Popek, and D. Rothmeier. "Implementation of the Ficus Replicated File System". In USENIX Summer' 90, pages 63-71, 1990.

[5] R. G.Guy, T. W. Page, J. S. Heidemann, and G. J. Popek. "Name Transparency in Very Large Scale Distributed File System". In Proc. of 2nd IEEE Workshop on Experiental Distributed Sys. tem, pages 20-25, Oct 1990.

[6] J. Ousterhout, A. Cherenson, F. Douglis, M. Nelson, and B. Welch. "The Sprite Network Operating System". IEEE Computer, 21(2):23-36, Feb. 1988. 
[7] M. Satyanarayanan. "Scalable, Secure, and Highly Available Distributed File Access". IEEE Compuser, 23(5):9-21, May 1990.

[8] A. Siegel, K. Birman, and K. Marzullo. "Deceit: A Flexible Distributed File System". In USENIX Summer '90, pages 51-61, 1990.

[9] M. G. Baker, J. H. Hartman, M. D. Kupfer, K. W. Shirriff, and J. Ousterhout. "Measurements of a Distributed File System". In Proc. of th Symposium Operating Systems Principles, pages 198212. ACM, 1991. 